${ }^{1}$ School of Psychiatry, University of Western Australia, and Department of Psychiatry, Fremantle Hospital, Fremantle, Western Australia, Australia;

${ }^{2}$ Department of Psychiatry, Maastricht University Hospital, Maastricht, The Netherlands

Correspondence to: Professor S E Starkstein, Education Building T-7.

Fremantle Hospital, Fremantle, 6959 WA, Australia; ses@cyllene.uwa.edu.au

Received 9 October 2007 Revised 27 November 2007

Accepted 1 December 2007

Published Online First

10 January 2008

\title{
The nosological position of apathy in clinical practice
}

\author{
S E Starkstein, ${ }^{1}$ A F G Leentjens ${ }^{2}$
}

\section{ABSTRACT}

Apathy is increasingly recognised as a common behavioural syndrome in psychiatric disorders, but it is conceptually ill defined. The aim of this study was to examine the concept of apathy as it is currently used in neurology and psychiatry, by review of the literature and conceptual analysis. There is no consensus on diagnostic criteria for apathy as a syndrome. Apathy is mostly defined as a disorder of motivation, and operationalised as diminished goal oriented behaviour and cognition. There is discussion about whether an emotional dimension should form part of the definition of apathy. Abulia is considered a more severe type of apathy, but its nosological position is still unclear. A structured clinical interview and a proposal for diagnostic criteria for apathy in dementia have been recently validated. There are several valid and reliable scales to measure the severity of apathy in patients with psychiatric and neurological disorders. In summary, apathy is increasingly recognised as a common behavioural syndrome associated with neuropsychiatric disorders. There is a need for consensus on diagnostic criteria to facilitate future research. From a nosological perspective, future studies should examine the overlap with other psychiatric and neurodegenerative conditions and further validate specific diagnostic and assessment tools.

In spite of a lack of a proper definition, "apathy" is increasingly diagnosed in patients with neurological and psychiatric conditions. There is however no consensus as to whether apathy should be considered a symptom of major neuropsychiatric disorders, or as a syndrome in its own right. In addition, critical questions regarding the phenomenology of apathy and the syndromal validity of this construct, as well as the relationship between apathy and similar concepts, such as abulia, the autoactivation deficit, athymormia, the negative syndrome and psychic akinesia, still remain unanswered. These important nosological problems, as well as issues related to the assessment of apathy, will be the focus of the present review article. Other relevant issues, such as epidemiology, demographic and clinical correlates, and putative mechanism of apathy in specific neuropsychiatric conditions, will not be directly addressed.

\section{THE HISTORY OF APATHY}

More than 2000 years ago the Greek philosophers of the Stoic school wrote that being apathěs - that is, free from passions-was the only road to a virtuous and happy life. ${ }^{1}$ They considered that states of extreme emotions would preclude clear thinking, thus inclining humans towards irrational behaviours. As rationality was considered the main human attribute, passions ought to be banished. During the Renaissance the term "apathy" was used in its ancient meaning (ie, as freedom from passions) by all the great humanists, from Erasmus to Vives. By the early 19th century however, the term apathy was mainly used to refer to states of "non-reactivity", both psychological and physical, as reflected in the condition of "adynamic stupor". ${ }^{2}$ These states were variably termed "apathie affective", "apathie intellectuelle" and "Unempfindlichkeit" (G Berrios, personal communication). The contemporary concept of apathy could not be more different than the Stoic. Rather than the preferred human lifestyle, apathy is now considered a defective behavioural state. In recent years, Marin has rekindled the interest in apathy by proposing a specific set of diagnostic criteria and validating ad hoc scales. ${ }^{34}$

\section{CURRENT DEFINITIONS OF APATHY}

Current psychiatric classification systems do not provide a definition of apathy. The Diagnostic and Statistical Manual of Mental Disorders, 4th Edn $(\mathrm{DSM}-\mathrm{IV})^{5}$ uses the term "apathy" only in the context of a specific subtype of personality change due to a general medical condition. The International Classification of Diseases (ICD)-10 makes no reference to apathy. ${ }^{6}$ In 1991, Marin proposed to define apathy as a distinct psychiatric syndrome, characterised by deficits in goal directed behaviours as manifested by the "simultaneous diminution in the cognitive and emotional concomitants of goal directed behaviour". ${ }^{3}$ He defined motivation as "the direction, intensity and persistence of goal directed behaviour", and identified loss of motivation as the main symptom of apathy. ${ }^{3}$ Marin structured the clinical expression of apathy around the concepts of (1) reduced goal directed behaviour, (2) reduced goal directed cognition and (3) reduced emotional concomitants of goal directed behaviours. Reduced goal directed behaviour was operationalised as lack of effort, initiative and productivity; reduced goal directed cognition was operationalised as decreased interests, lack of plans and goals, and lack of concern about one's own health or functional status; and finally, reduced emotional concomitants of goal directed behaviours were operationalised as flattened affect, emotional indifference and restricted responses to important life events. ${ }^{3}$ This construct was slightly modified and organised into a standardised set of diagnostic criteria by Starkstein (box 1). ${ }^{7}$ A similar division of apathy into emotional, cognitive and behavioural domains was more recently proposed by van Reekum and colleagues. $^{8}$ According to Marin and Wilkosz, ${ }^{9}$ apathy should be considered the expression of diminished motivation, but stated that patients with apathy "are generally able to initiate and sustain behaviour, describe their plans, goals and 
interests, and react emotionally to significant events and experiences", although these features are less evident than among individuals without apathy.

Rarely has apathy been defined based on the more historically appropriate concept of disturbance of emotions. Sims defined apathy as the absence of feeling that frequently occurs together with blunting (defined as "lack of emotional sensitivity") and flattening of affective response (defined as a restriction in the usual range of emotional display). ${ }^{10}$ This definition is conceptually related to the construct of apathy as a disorder of feeling and/or expression of emotions, whereas Marin's definition is conceptually related to the construct of apathy as a disorder of drive and motivation. ${ }^{9}$

Levy and Dubois recently suggested that apathy should not be defined as lack of motivation, on the basis that such a definition would be a psychological interpretation of a behavioural change. ${ }^{11}$ Instead, they defined apathy as an observable behavioural syndrome consisting of a quantitative reduction of self-generated voluntary and purposeful behaviours. They further stressed that the extent of this behavioural change is relative to the previous behavioural pattern of the individual, should occur in the absence of contextual or physical changes, and should be reversed by external stimulation.

Summarising, there is no consensus on whether apathy should be considered primarily a disorder of drive and motivation, a disorder of the (expression of) emotions, or both. At present, the most widely used definitions of apathy include behavioural and cognitive dimensions of apathy, but opinions differ on the inclusion of an emotional dimension.

\section{APATHY AS A SYNDROME}

In the current literature, discussion focuses on whether apathy is a symptom of psychiatric disorders, such as dementia or depression, or whether it is a syndrome in its own right. Moreover, these two alternatives need not be mutually

Box 1 Diagnostic criteria for apathy (Starkstein 2000', adapted from Marin 1991 ${ }^{32}$ ) (adaptations proposed by the authors are indicated in bold type)

(A) Lack of motivation relative to the patient's previous level of functioning or the standards of his or her age and culture as indicated either by subjective account or observation by others.

(B) Presence for at least 4 weeks during most of the day, of at least 1 symptom belonging to each of the following three domains:

Diminished goal directed behaviour

1. Lack of effort or energy to perform everyday activities.

2. Dependency on prompts from others to structure everyday activities.

Diminished goal directed cognition

1. Lack of interest in learning new things, or in new experiences.

2. Lack of concern about one's personal problems.

Diminished concomitants of goal directed behaviour

1. Unchanging or flat affect

2. Lack of emotional responsivity to positive or negative events.

(C) The symptoms cause clinically significant distress or impairment in social, occupational or other important areas of functioning.

(D) The symptoms are not due to diminished level of consciousness or the direct physiological effects of a substance. exclusive. In dementia, some view apathy as an intrinsic symptom of cognitive deterioration. ${ }^{3}$ On the other hand, most studies showed that approximately $50 \%$ of patients with dementia do not develop apathy. ${ }^{12} 13$

Poor motivation is a cardinal diagnostic symptom of depression, which makes it difficult to separate apathy from depression. The frequent overlap between depression and apathy is based on the conceptual fact that loss of interest/ motivation is a key criterion for both syndromes.

\section{Cognitive deterioration and dementia}

Marin suggested that apathy should not be diagnosed in the context of diminished level of consciousness, moderate or severe cognitive deficits, or marked emotional distress. This exclusion was made on the basis that apathy may be an intrinsic symptom of dementia: "...in Alzheimer's disease, apathy tends to occur in the confusional state of the disease". "Whereas this strategy could help to identify a syndrome of "pure" apathy, uncontaminated by changes in cognitive or affective domains, several conceptual and empirical findings argue against this approach. A relevant conceptual issue is that a syndrome is defined as a constellation of symptoms, without reference to a specific aetiology. Therefore, it is unclear why apathy should be considered a syndrome in some contexts (eg, stroke), but not in others (eg, dementia). Moreover, it is known that some psychopathological syndromes, such as depression and apathy, usually occur together in neurodegenerative diseases. ${ }^{14}$ In fact, the syndrome of apathy is most frequent among individuals with neurological disorders and some degree of cognitive impairment and depression, such as Alzheimer's disease, stroke or Parkinson's disease. ${ }^{15-17}$ Several studies in patients with dementia showed a significant association between executive dysfunction and more severe apathy, although this finding could not be consistently replicated. ${ }^{8}$ Cognitive deficits are not sufficient to produce apathy, given that in some studies about half of the individuals with moderate or severe dementia did not suffer from apathy. ${ }^{18} 19$ Whether cognitive deficits are necessary to produce apathy has not been specifically examined, although most studies assessing apathy in a variety of neurological disorders reported relatively low scores on the Mini-Mental State Examination for patients with apathy. ${ }^{8}$ Whether the syndrome of apathy associated with overt dementia has a different phenomenology than the syndrome of apathy in other neurological disorders (eg, stroke, traumatic brain injury, Parkinson's disease or frontal lobe lesions) or psychiatric disorders (eg, depression or schizophrenia) has not been examined.

\section{Depression}

The DSM-IV allows the diagnosis of depression in the absence of a depressed mood provided that symptoms of loss of interest or anhedonia are present. Similarly, the ICD-10 states that patients with loss of interest but no depressed mood may still qualify for a diagnosis of mild or moderate depression provided they suffer from decreased energy. This definition may explain the high frequency of co-occurrence of depression and apathy. The question arises as to whether these non-dysphoric depressions can be reliably separated from apathy, and whether lack of interest and/or anhedonia are core symptoms of diminished motivation.

Lack of interest is one of the diagnostic criteria used to operationalise the "diminished goal directed cognition" dimension of apathy (see box 1). Anhedonia is defined as the 
(complete) inability to experience pleasure, as manifested in facial expression, speech, behaviour, lifestyle and the individual's account of personal experience. ${ }^{10}$ The nosological relationship between apathy and anhedonia depends on how apathy is conceptualised. If apathy is considered a state of absence of feeling and emotional sensitivity, anhedonia should be considered a mandatory symptom of apathy. If, on the other hand, apathy is considered a state of diminished motivation, anhedonia may not be a necessary diagnostic criterion. A preliminary study of apathy in dementia showed that patients with apathy but no depression rarely report anhedonia, suggesting that anhedonia is more characteristic of depression than apathy (SE Starkstein, personal communication).

Apathy has been associated with depression in a number of studies. In Parkinson's disease the combination of apathy and depression was reported to be more frequent than depression without apathy, ${ }^{20}$ although other studies reported that apathy in the absence of depression is actually more common than their association. $^{21} 22$ Apathy without depression has also been consistently found in Alzheimer's disease, frontotemporal dementia, progressive supranuclear palsy and stroke. ${ }^{23} 24$

\section{DIAGNOSING APATHY}

Marin and Wilkosz suggested that apathy should be diagnosed only after a comprehensive neuropsychiatric evaluation, including the assessment of the individual's social and physical environment. ${ }^{9}$ They further stressed the importance of taking into consideration the great variability in each individual's goals, interests, emotional displays and activities, all of which are strongly influenced by general experience, education, social class, age cohort and a host of other cultural factors. All of these considerations are certainly relevant and should be explicitly considered for any clinical assessment of apathy. The dilemma is how to formally diagnose apathy given the lack of diagnostic criteria and paucity of standardised instruments.

\section{Diagnostic criteria}

An adequate diagnosis requires reliable and valid diagnostic criteria. To date there are no diagnostic criteria for apathy that are endorsed by psychiatric nomenclatures or scientific societies. The authors strongly stress the importance of consensus on such criteria in order to facilitate future research. At present, the only criteria available are those formulated by Marin, which were later adapted by the first author ${ }^{25}$ (see box 1). This adaptation not only included a restructuring and rephrasing of the original criteria, but also abandoned the requirement of no intellectual decline. Using these reformulated criteria, apathy was diagnosed in $37 \%$ of 319 patients with dementia, in $32 \%$ of 157 patients with depression but no dementia and in none of 36 age comparable healthy individuals. ${ }^{19}$ While these criteria have been used in several recent research studies, ${ }^{13}{ }^{14}$ they still lack the requirement for consistency of the symptoms over time, as well as a defined time frame. We therefore propose a second adaptation that requires the symptoms to be present for at least 4 weeks during most of the day (box 1).

Marin stressed the apparent contradiction of diagnosing apathy among patients with frontal lobe lesions, who may show features of both apathy and concurrent violent behaviour, implying that apathy and behavioural disinhibition are opposite expressions of a common behavioural domain. ${ }^{4}$ Nevertheless, several studies demonstrated a significant association between apathy and disinhibition in patients with dementia, ${ }^{12}{ }^{26}$ suggesting that apathy and disinhibition may alternate in the same individual. It is important to note that these patients show disinhibited behaviours only sporadically, while apathy is present most of the time.

\section{Diagnostic interviews}

The mental status examination in neuropsychiatry research should be assessed using semi-structured interviews, such as the Schedules for Clinical Assessment in Neuropsychiatry ${ }^{27}$ or the Structured Clinical Interview for DSM-IV ${ }^{28}$ The problem with apathy is that none of the structured psychiatric interviews include sections with specific questions for this condition. The first author has recently published the validation of the Structured Clinical Interview for Apathy to diagnose apathy in dementia. This instrument showed adequate construct validity, strong internal consistency, and high test-retest and inter-rater reliability. ${ }^{29}$

\section{Assessment scales}

Several instruments are currently used to measure the severity of apathy in neurological and psychiatric disorders. Marin et al developed the Apathy Evaluation Scale, an 18 item scale that can be administered as a self-rated scale, as a caregiver paper and pencil test or as a clinician administered test. ${ }^{30}{ }^{31}$ This scale was validated in patients with Alzheimer's disease and other dementias, stroke and major depression ${ }^{32}{ }^{33}$ The Apathy Scale was developed as an abridged and modified version of Marin's scale, and validated in patients with Parkinson's disease. ${ }^{16} 17$ Subsequently, the scale has also been used in patients with stroke $^{15}$ and Alzheimer's disease. ${ }^{19}$ The Children's Motivation Scale is also based on the Apathy Evaluation Scale, and rates the severity of apathy in children and adolescents. ${ }^{3}$ Strauss and Sperry developed the Dementia Apathy Interview and Rating to assess dementia related changes in motivation, emotional responsiveness and engagement. ${ }^{34}$ The interviewer scores the frequency of specific behaviours over the past month on the basis of responses provided by the primary caregiver, with further clarifications as to whether the given behaviour has changed from the time prior to dementia onset. Robert et al designed the Apathy Inventory as a rating scale for the global assessment of apathy, and with separate assessments for emotional blunting, lack of initiative and loss of interest. It was validated in Alzheimer's disease, mild cognitive impairment and Parkinson's disease. ${ }^{35}$ Recently, the Lille Apathy Rating Scale was validated for use in Parkinson's disease. ${ }^{36}$ Apathy is also assessed as a specific domain of the Neuropsychiatric Inventory, a multidimensional instrument that is administered to caregivers. ${ }^{37}$

\section{THE DIFFERENTIAL DIAGNOSIS OF APATHY}

Several neuropsychiatric syndromes, which are still regularly used in clinical practice or research, share one or more symptoms with the apathy syndrome, but the nosological relation of these syndromes to the concept of apathy remains uncertain. These syndromes include abulia, akinetic syndromes, despair and demoralisation, depression and the negative syndrome in schizophrenia, and are briefly discussed below.

\section{Abulia}

Ribot defined abulia as "loss, lack or impairment of the power of the will to execute what is in mind", and stressed the lack of muscular problems. ${ }^{38}$ Ribot considered that abulia was not the result of "lack of desire" but was the consequence of abnormalities in "the transition from motive and desire, to 
execution" due to low activation or dysfunction of the "motor centres". In contrast, Marin defined abulia as a "lack of will or motivation, or an inability to decide", and considered abulia and apathy to be on a continuum of motivational and emotional deficit, with abulia as the most severe manifestation. ${ }^{4}$ Marin and Wilkosz suggested that the symptoms of abulia include poverty of behaviour and speech output, lack of initiative, loss of emotional responses, psychomotor slowing and prolonged speech latency. ${ }^{9}$ Bhatia and Marsden defined abulia as "apathy with loss of initiative and of spontaneous thought and emotional responses", 39 and found this syndrome to be related to lesions of the basal ganglia. In a study that focused on behavioural changes after basal ganglia lesions, Giroud et al defined abulia as "the loss of mental and motor initiative and drive...inducing apathy and blunting of responses". ${ }^{40}$ Vijayaraghavan et al assessed the concept of abulia in a group of psychiatric and neurological experts. ${ }^{41}$ The final consensus was that the following eight features should be considered typical of abulia: (1) difficulty to initiate and sustain purposeful movements; (2) poverty of spontaneous movements; (3) reduced spontaneous speech; (4) increased response time to queries; (5) passivity; (6) reduced emotional responsiveness and spontaneity; (7) reduced social interaction; and (8) reduced interest in usual pastimes. Most participants acknowledged that the terms apathy and abulia were often used interchangeably in clinical practice.

\section{Akinetic syndromes}

Psychic akinesia has been described in patients that are fully responsive, but with no display of goal directed activities. It is characteristic of patients with "psychic akinesia" to correctly perform most of their daily activities, but only after strong stimulation. ${ }^{42}$ A similar syndrome was labelled the "autoactivation deficit" by Laplane and Dubois, and was defined as "a deficit in spontaneous activation of mental processing, observed in behavioural, cognitive or affective domains, which can be totally reversed by external stimulation that activates normal patterns of response". ${ }^{43}$ The authors listed the following symptoms of autoactivation deficit: (1) inertia (defined as the tendency to stay immobile for long periods and taking no initiatives), (2) mental emptiness (defined as lack of thoughts and no projections onto the future), (3) repetitive and stereotyped activities, (4) flat affect and (5) blunted emotional responses. Full reversal of the negative state on external stimulation was considered the main clinical difference between the autoactivation deficit and abulia. On the other hand, the criteria for "full" or "partial" reversal were not spelled out and the construct of the "autoactivation deficit" has not been validated. As indicated above, the concept of abulia is not well defined, and to separate the lesser known syndrome of autoactivation deficit from abulia may be hard to accomplish in a reliable way.

A syndrome similar to the autoactivation deficit has been recently described by Habib under the term "athymormia", from the Greek words thumos (mood) and horme (impulse). ${ }^{44}$ This syndrome was given the following clinical description: "a striking reduction in spontaneous motion and speech, with subjacent "mental emptiness", a loss of interest for previously motivating activities and an apparent flatness or at least poor expressiveness of affect". ${ }^{44}$ Habib further suggested that this syndrome occurs in the absence of underlying depression or "pathology of action", and that the cause should be sought at the "emotional-limbic" level.
Sims defined akinetic mutism as the inability to initiate action in a patient who appears alert. ${ }^{10}$ Akinetic mutism is described in the Structured Clinical Assessment in Neuropsychiatry as a state of immobility due to lack of voluntary movement, mutism or the articulation of monosyllables, and vigilant gaze. Marin and Wilkosz ${ }^{9}$ defined akinetic mutism as the total absence of spontaneous behaviour and speech, occurring in the presence of preserved visual tracking. These authors proposed the term "disorders of diminished motivation" to include akinetic mutism, abulia and apathy within a single nosological domain. They considered these disorders of diminished motivation to lie on a continuum, with apathy as the less severe manifestation of diminished motivation and akinetic mutism as the most severe.

\section{Despair and demoralisation}

This is considered a psychological state occurring primarily in normal individuals in response to the experience of overwhelming stress or important changes in their personal and/or social environment. ${ }^{4}$ Despair and demoralisation share with apathy the symptom of loss of motivation, but whereas apathy also denotes a lack of concern about the present and future, despair and demoralisation are experienced as unpleasant states with an affective orientation towards the future. ${ }^{4}$

\section{The negative syndrome}

Apathy has long been recognised as a common behavioural change in schizophrenia. Back in 1911, Bleuler described "an indifference to everything - to friends and relations, to vocation or enjoyment, to duties or rights, to good fortune or to bad". ${ }^{45}$ Kraepelin noted that his patients had lost "every independent inclination for work and action; they sit about idle, trouble themselves about nothing, do not go to their work, neglect their most pressing obligations, although they are perhaps still capable of employing themselves in a reasonable way if stimulated from outside". ${ }^{46}$ More recently, Kiang et al showed that apathy is not significantly related to either the positive symptoms of schizophrenia or to depressive symptoms, suggesting that apathy is an independent behavioural domain. ${ }^{47}$ They also found that apathy was the strongest predictor of poor functional outcome in schizophrenia.

Patients with schizophrenia are aware of their increased apathy, and also identify apathy as the most bothersome symptom..$^{48}$ Symptoms of apathy such as restricted affect, low social drive and poverty of speech may be caused by multiple factors, such as neuroleptic drugs, the psychotic experience itself (eg, being unable to work because of auditory hallucinations or paranoid delusions), relevant contextual factors (eg, poor social support) or a combination of these. Taken together, clinical descriptions and empirical studies on the negative syndrome of schizophrenia suggest that apathy may be a criterion, perhaps a key one, of this syndrome. On the other hand, the negative syndrome of schizophrenia has more clinical complexity than apathy, both in terms of its phenomenology and putative mechanism.

\section{GENERAL CONCLUSION}

The term apathy is currently used to refer to disorders of diminished motivation, as manifested by reduced goal oriented behaviours and cognitions. There is no agreement on whether emotional deficits are part of apathy. Studies into the phenomenology of apathy are hampered by the lack of consensus on diagnostic criteria. Several rating scales have been 
developed over the past years to measure the severity of apathy, but proper validation is limited by the lack of standard criteria. The nosological relation of apathy with a number of other syndromes that share one or more symptoms with apathy is uncertain. Neither the diagnostic reliability nor the clinical validity of these putative syndromes has been demonstrated. While apathy frequently occurs together with depression and cognitive decline, it is also present in patients with neither depression nor cognitive deficits. Marin proposed that abulia should be considered a more severe type of apathy, and akinetic mutism as the most severe condition within the domain of disorders of diminished motivation. ${ }^{9}$ The negative syndrome of schizophrenia has apathy as one of its main criteria, but consists of a more complex phenomenology. Future studies should address the validity of apathy as a distinct syndrome and examine its phenomenological presentation in different neurological and psychiatric diseases, the mechanism of apathy in these disorders and its long term clinical impact.

The treatment of apathy remains a challenge. Several studies suggest that cholinesterase inhibitors may be useful to treat apathy in dementia, although the results were not specific for apathy. ${ }^{49}$ Dopaminergic agonists and psychostimulants have shown some efficacy to treat apathy in a variety of neurological conditions but these studies were mostly limited to case reports or small open label trials. ${ }^{50-53}$ Consensus on diagnostic criteria would open the door for randomised controlled treatment studies that may reveal possibilities to influence the behavioural, cognitive and emotional concomitants of apathy in neurological and psychiatric disorders.

Funding: The study was partially supported by grants from the National Health and Medical Research Council of Australia.

Competing interests: None.

\section{REFERENCES}

1. Inwood B. Ethics and human action in early stoicism. Oxford: Clarendon Press, 1985.

2. Berrios GE. The history of mental symptoms. Cambridge: Cambridge University Press, 1996.

3. Marin RS. Apathy: a neuropsychiatric syndrome. J Neuropsychiatry Clin Neurosci 1991:3:243-54.

4. Marin RS. Differential diagnosis and classification of apathy. Am J Psychiatry 1990; 147:22-30

5. American Psychiatric Association. Diagnostic and statistical manual of mental disorders, 4th Edn. Washington, DC: American Psychiatric Press, 1994.

6. World Health Organization. The ICD-10 Classification of Mental and Behavioural Disorders. Geneva: WHO, 1993.

7. Starkstein SE. Apathy and withdrawal. Int Psychogeriatr 2000;12(Suppl 1):135-8.

8. van Reekum R, Stuss DT, Ostrander L. Apathy: why care? J Neuropsychiatry Clin Neurosci 2005;17:7-19.

9. Marin RS, Wilkosz PA. Disorders of diminished motivation. J Head Trauma Rehabil 2005;20:377-88.

10. Sims A. Symptoms in the mind. London: Saunders, 2003.

11. Levy R, Dubois B. Apathy and the functional anatomy of the prefrontal cortex-basal ganglia circuits. Cerebral Cortex 2006;16:916-28.

12. Levy ML, Cummings JL, Fairbanks LA, et al. Apathy is not depression. J Neuropsychiatry Clin Neurosci 1998;10:314-19.

13. Starkstein SE, Ingram L, Garau ML, et al. On the overlap between apathy and depression in dementia. J Neuropsychiatry Clin Neurosci 2005;76:1070-4.

14. Starkstein SE, Jorge R, Mizrahi R, et al. A prospective longitudinal study of apathy in Alzheimer's disease. J Neurol Neurosurg Psychiatry 2006;77:8-11.

15. Starkstein SE, Fedoroff JP, Price TR, et al. Apathy following cerebrovascular lesions. Stroke 1993;24:1625-30.

16. Starkstein SE, Mayberg HS, Preziosi TJ, et al. Reliability, validity, and clinica correlates of apathy in Parkinson's disease. J Neuropsychiatry Clin Neurosci 1992:4:134-9.
17. Starkstein SE, Migliorelli R, Manes F, et al. The prevalence and clinical correlates of apathy and irritability in Alzheimer's disease. Eur J Neurol 1995;2:540-6.

18. Starkstein SE, Merello M. Psychiatric and cognitive disorders in Parkinson's disease. Cambridge: Cambridge University Press, 2002.

19. Starkstein SE, Petracca G, Chemerinski E, et al. Syndromic validity of apathy in Alzheimer's disease. Am J Psychiatry 2001;158:872-7.

20. Dujardin K, Sockeel P, Devos D, et al. Characteristics of apathy in Parkinson's disease. Mov Disord 2007;22:778-84.

21. Kirsch-Darrow L, Fernandez HH, Marsiske $\mathrm{M}$, et al. Dissociating apathy and depression in Parkinson's disease. Neurology 2006;67:33-8.

22. Isella V, Melzi P, Grimaldi $M$, et al. Clinical, neuropsychological, and morphometric correlates of apathy in Parkinson's disease. Mov Disord 2002;17:366-71.

23. Levy ML, Cummings J, Fairbanks L, et al. Apathy is not depression. J Neuropsychiatry Clin Neurosci 1998;10:314-19.

24. Starkstein SE, Ingram L, Garau ML, et al. On the overlap between apathy and depression in dementia. J Neurol Neurosurg Psychiatry 2005;76:1070-4.

25. Marin RS. Apathy, a neuropsychiatric syndrome. J Neuropsychiatry Clin Neurosci 1991;3:243-54.

26. Starkstein SE, Garau ML, Cao A. Prevalence and clinical correlates of disinhibition in dementia. Cogn Behav Neurol 2004;17:139-47.

27. World Health Organization. Schedules for clinical assessment in neuropsychiatry V.2.0. Geneva: World Health Organization, 1994.

28. Spitzer RL, Williams JB, Gibbon M, et al. The Structured Clinical Interview for DSMIII-R (SCID). I: History, rationale, and description. Arch Gen Psychiatry 1992;49:624-9.

29. Starkstein SE, Ingram L, Garau LM, et al. On the overlap between apathy and depression in dementia. J Neurol Neurosurg Psychiatry 2005;76:1070-4.

30. Marin RS, Butters MA, Mulsant BH, et al. Apathy and executive function in depressed elderly. Journal Geriatr Psychiatry Neurol 2003;16:112-16.

31. Marin RS, Firinciogullari S, Biedrzycki RC. Group differences in the relationship between apathy and depression. J Nerv Ment Dis 1994;182:235-9.

32. Marin RS, Biedrycki RC, Firiciogullari S. Reliability and validity of the Apathy Evaluation Scale. Psychiatry Res 1991;38:2.

33. Pluck GC, Brown RG. Apathy in Parkinson's disease. J Neurol Neurosurg Psychiatry 2002:73:636-42.

34. Strauss ME, Sperry SD. An informant-based assessment of apathy in Alzheimer disease. Neuropsychiatry Neuropsychol Behav Neurol 2002;15:176-83.

35. Robert PH, Clairet S, Benoit $\mathrm{M}$, et al. The apathy inventory: assessment of apathy and awareness in Alzheimer's disease, Parkinson's disease and mild cognitive impairment. Int J Geriatr Psychiatry 2002;17:1099-105.

36. Sockeel P, Dujardin K, Devos D, et al. The Lille apathy rating scale (LARS), a new instrument for detecting and quantifying apathy: validation in Parkinson's disease. J Neurol Neurosurg Psychiatry 2006;77:579-84.

37. Cummings JL. The Neuropsychiatric Inventory: Assessing psychopathology in dementia patients. Neurology 1997; $\mathbf{4 8}($ Suppl 6):S10-16

38. Ribot TH. Les Malades de la Volonté, 18th Edn. Paris: Alcan, 1904.

39. Bhatia KP, Marsden CD. The behavioural and motor consequences of focal lesions of the basal ganglia in man. Brain 1994;117:859-76.

40. Giroud M, Lemesle M, Madinier G, et al. Unilateral lenticular infarcts: radiological and clinical syndromes, aetiology, and prognosis. J Neurol Neurosurg Psychiatry 1997;63:611-15.

41. Vijayaraghavan L, Krishnamoorthy ES, Brown RG, et al. Abulia: a delphi survey of British neurologists and psychiatrists. Mov Disord 2002;17:1052-7.

42. Starkstein SE, Berthier M, Leiguarda R. Psychic akinesia following bilateral pallidal lesions. Int J Psychiatry Med 1989;19:155-64.

43. Laplane D, Dubois B. Auto-activation deficit: a basal ganglia related syndrome. Mov Disord 2001;16:810-14.

44. Habib M. Athymhormia and disorders of motivation in basal ganglia disease. J Neuropsychiatry Clin Neurosci 2004;16:509-24.

45. Bleuler E. Dementia praecox, or the group of schizophrenias. New York: International Universities Press, 1911.

46. Kraepelin E. Dementia praecox and paraphrenia. Edinburgh: ES Livingstone, 1919

47. Kiang M, Christensen BK, Remington G, et al. Apathy in schizophrenia: clinical correlates and association with functional outcome. Schizophr Res 2003;63:79-88.

48. Selten JP, Wiersma D, van den Bosch RJ. Distress attributed to negative symptoms in schizophrenia. Schizophr Bull 2000;26:737-44.

49. Boyle PA, Malloy PF. Treating apathy in Alzheimer's disease. Dement Geriatr Cogn Disord 2004;17:91-9.

50. Chatterjee A, Fahn S. Methylphenidate treats apathy in Parkinson's disease. J Neuropsychiatry Clin Neurosci 2002;14:461-2.

51. Padala PR, Burke WJ, Bhatia SC, et al. Treatment of apathy with methylphenidate J Neuropsychiatry Clin Neurosci 2007:19:81-3.

52. Marin RS, Fogel BS, Hawkins J, et al. Apathy: a treatable syndrome. J Neuropsychiatry Clin Neurosci 1995;7:23-30.

53. Corcoran C, Wong ML, O'Keane V. Bupropion in the management of apathy. J Psychopharmacol 2004;18:133-5. 